\title{
Light Particle Emission Mechanisms in Heavy-lon Reactions at 5-20 MeV/u
}

\author{
V.L. Kravchuk ${ }^{1, a}$, S. Barlini² ${ }^{2}$ and O.V. Fotina ${ }^{3}$ for the NUCL-EX - HECTOR collaboration \\ 1 INFN, Laboratori Nazionali di Legnaro, I-35020 Legnaro (Padova), Italy and \\ Dipartimento di Fisica dell'Università di Bologna, I-40126 Bologna, Italy \\ 2 INFN, Sezione di Firenze, I-50125 Firenze, Italy \\ 3 Skobeltsyn Institute of Nuclear Physics, Moscow State University, 119992 Moscow, Russia
}

\begin{abstract}
Light Charged Particle emission mechanisms were studied for different mass entrance channel nuclear reactions. The $300,400,500 \mathrm{MeV}{ }^{64} \mathrm{Ni}+{ }^{68} \mathrm{Zn}$ and $130,250 \mathrm{MeV}{ }^{16} \mathrm{O}+{ }^{116} \mathrm{Sn}$ reactions were measured at the Legnaro National Laboratory using the beams from the TANDEM-ALPI acceleration system. Light Charged Particles were measured in coincidence with Evaporation Residues and their spectra were analyzed using the global moving source fit technique. The characterization of different emission sources (evaporative, pre-equilibrium, break-up) is discussed. The behavior of pre-equilibrium emission as a function of projectile energy, excitation energy and mass-asymmetry in the entrance channel was studied, evaluating the energy, mass and charge lost by the composite systems and using Griffin exciton model for the pre-equilibrium neutron emission. The present results are compared with the systematics of the asymmetric mass entrance channel reactions. The present work shows that also at the onset the pre-equilibrium emission depends not only on the projectile velocity but also on the reaction entrance channel mass-asymmetry. The first attempt of the particle spectra analysis using the Griffin exciton model is demonstrated for the case of proton emission in the $130 \mathrm{MeV}{ }^{16} \mathrm{O}+{ }^{116} \mathrm{Sn}$ reaction.
\end{abstract}

\section{Introduction}

Low energy nuclear reactions have been extensively studied in the past, devoting mainly the attention to evaporation of light particles from thermalized systems [1,2]. The problem of different emissions, before a complete thermalization could occur, shows up as soon as the bombarding energy grows up, especially approaching $10 \mathrm{MeV} / \mathrm{u}$. A certain progress has been achieved in the understanding of pre-equilibrium emission of Light Charged Particles (LCP) [3-5] and neutrons [5-8] during the past two decades. Nevertheless, the knowledge on the fast emission processes, showing a dependence on both the entrance channel massasymmetry and on the beam velocity, is far from being complete. The pre-equilibrium process plays an important role on the dynamics of heavy ion fusion reactions in the early stages and also characterizes the features of the hot fused source.

The LCP pre-equilibrium process becomes evident around a beam energy of $10 \mathrm{MeV} / \mathrm{u}$. Since the fast emission remembers the entrance channel, the final expected distribution is influenced by the velocity of the fast emitting source and will be, therefore, forwardely peaked with a shape which is strongly dependent on the reaction dynamics. It should be noted that the existing experiments mainly employed a small number of detectors (with small solid angle coverage), and thus the high-energy tails of the measured distribution are characterized by low statistics. Looking at the status of the art on this subject it is obvious

\footnotetext{
a e-mail: kravchuk@lnl.infn.it
}

that further and more complete information is needed to develop a coherent framework of the pre-equilibrium processes. Many modern new generation $4 \pi$ apparata are nowdays available which allow highly efficient coincidence measurements. A major reconstruction of the whole angular distribution can be reached with a better discrimination between equilibrium and non-equilibrium emitted particles. A high statistics can be collected which is especially important to study the high-energy part of the emission spectra and can provide a larger sensitivity for the discrimination of different emission mechanisms. Furthermore a severe selection of the events performed through opportune triggering coincidences can strongly help in avoiding unwanted competing mechanisms. All these issues have motivated new measurements to study the LCP emission mechanisms from several nuclear reactions as a function of different mass asymmetries in the entrance channel and different bombarding energies.

We performed a series of experiments employing the GARFIELD (General ARray for Fragment Identification and for Emitted Light particles in Dissipative collisions) apparatus [9] at the Laboratori Nazionali di Legnaro. The LCP were measured in coincidence with Evaporation Residues (ER). In this paper we report on the results concerning protons and $\alpha$ particles emission for the mass-symmetric entrance channel reactions $300,400,500 \mathrm{MeV}{ }^{64} \mathrm{Ni}+{ }^{68} \mathrm{Zn}$ and the mass-asymmetric entrance channel reactions 130 , $250 \mathrm{MeV}{ }^{16} \mathrm{O}+{ }^{116} \mathrm{Sn}$. In case of complete fusion they would all lead to the Compound Nucleus ${ }^{132} \mathrm{Ce}^{*}$, with excitation energies of $100,150,200 \mathrm{MeV}$ in the $\mathrm{Ni}$ case and

This is an Open Access article distributed under the terms of the Creative Commons Attribution-Noncommercial License, which permits unrestricted use, distribution, and reproduction in any noncommercial medium, provided the original work is properly cited. 
$100,200 \mathrm{MeV}$ for the $\mathrm{O}$ induced reactions, respectively. The experimentally obtained spectra were analyzed using the golobal moving source fit technique. The model for the simultaneous description of the equilibrium and nonequilibrium processes recently developed on the basis of statistical theory of nuclear reactions and Griffin exciton model was applied to describe experimental results for a single case of protons emission in the $130 \mathrm{MeV}{ }^{16} \mathrm{O}+$ ${ }^{116} \mathrm{Sn}$ reaction.

\section{Experimental Setup}

The measurements were performed in the third experimental hall at the Legnaro National Laboratory. Pulsed beams (around $1 \mathrm{~ns}$ FWHM) of ${ }^{64} \mathrm{Ni}$ and ${ }^{16} \mathrm{O}$ provided by the TANDEM-ALPI acceleration system were used to bombard, respectively, self-supporting ${ }^{68} \mathrm{Zn}$ and ${ }^{116} \mathrm{Sn}$ targets, $500 \mu \mathrm{g} / \mathrm{cm}^{2}$ thick.

The LCP emitted either in fast processes or in slower evaporation decays were detected with the GARFIELD apparatus. The GARFIELD array was specially designed to study nuclear processes in the low and intermediate energy range $(5-20 \mathrm{MeV} / \mathrm{u})$ heavy-ion induced reactions. The operation of the GARFIELD apparatus largely bases on the $\triangle \mathrm{E}-\mathrm{E}$ technique, in which the $\triangle \mathrm{E}$ signal is given by the drift chamber, where gaseous micro-strip detectors collect and amplify the primary electrons produced along the ionization track of the detected particle. The CsI(Tl) scintillation detectors, lodged in the same gas volume, are used to get information on the residual energy E. The advantages of using micro-strip gas chambers are in the large dynamical range and high signal-to-noise ratio ( $\mathrm{Z}$ identification from low energy protons up to highly ionizing heavy ions). The GARFIELD drift chamber, filled with $\mathrm{CF}_{4}$ gas at low pressure ( $50 \mathrm{mbar}$ ), is azimuthally divided into 24 sectors, and each sector consists of $8 \Delta \mathrm{E}-\mathrm{E}$ telescopes, for a total of 192 telescopes covering an angular range from $\theta=29^{\circ}$ to $\theta=82^{\circ}$ and $2 \pi$ in $\phi$. The ER produced in the complete or incomplete fusion reactions under study have been detected using two couples of Position Sensitive Parallel Plate Avalanche Counters.

\section{Results and Discussion}

The choice of the reactions was dictated by the following purposes. The first one was to study the LCP emission mechanism from the composite systems formed with the same excitation energies $E^{*}{ }_{C N} \cong 100,200 \mathrm{MeV}$ but resulting from fusion reactions with different mass asymmetries in the entrance channel: the symmetric system ${ }^{64} \mathrm{Ni}+{ }^{68} \mathrm{Zn}$ at 300 and $500 \mathrm{MeV}$ and the asymmetric one ${ }^{16} \mathrm{O}+{ }^{116} \mathrm{Sn}$ at 130 and $250 \mathrm{MeV}$. The second purpose was to gain information on the LCP emission mechanism in reactions in which the same energy per nucleon is used for the projectile, but with different entrance channel mass asymmetries: $7.8 \mathrm{MeV} / \mathrm{u}\left(500 \mathrm{MeV}\right.$ total) ${ }^{64} \mathrm{Ni}+{ }^{68} \mathrm{Zn}$ and $8.1 \mathrm{MeV} / \mathrm{u}$ $(130 \mathrm{MeV}$ total $){ }^{16} \mathrm{O}+{ }^{116} \mathrm{Sn}$. Finally, the observation of the opening of the LCP pre-equilibrium emission channel in the case of mass-symmetric reactions between 6.3 $\mathrm{MeV} / \mathrm{u}\left(400 \mathrm{MeV}\right.$ total) ${ }^{64} \mathrm{Ni}+{ }^{68} \mathrm{Zn}$ and $7.8 \mathrm{MeV} / \mathrm{u}(500$ $\mathrm{MeV}$ total ${ }^{64} \mathrm{Ni}+{ }^{68} \mathrm{Zn}$ makes it interesting to continue the study of the symmetric case at even higher energies,

\subsection{Moving Source Analysis}

To describe the experimental LCP spectra, we employed the commonly used moving source analysis technique (see e.g. [3]). The moving source global fit allows to consider different sources of emitted particles, which give rise to different energy distributions. The strength of the global fit is that the resulting parameters have been obtained considering the whole experimental angular distribution covered by 192 detectors used in this work. Initially in our analysis we considered two thermal sources. The first (evaporative) source is based on the surface emission from the fully thermalized composite system and associated with the statistical particle distribution. The evaporative particle spectrum in the emitting source frame is represented by a Maxwellian distribution. The second (fast or pre-equilibrium) source we used is based on a volume emission. For the description of the pre-equilibrium contribution to the total experimental yield, we used the Watt distribution, which was successfully applied in previous works (see e.g. [3,5]) in order to fit an excessive yield of the total LCP spectra.

In Fig. 1 the result of the moving source fit for $\alpha$ particles for the $500 \mathrm{MeV}{ }^{64} \mathrm{Ni}+{ }^{68} \mathrm{Zn}$ reactions is shown. In this case the description of the experimental spectrum using only the evaporative source fit is reasonably good. The very small extra yield, which was not described by the evaporative source fit in the $500 \mathrm{MeV}{ }^{64} \mathrm{Ni}+{ }^{68} \mathrm{Zn}$ reaction gives a signature of a possible very small contribution coming from a pre-equilibrium $\alpha$-particle emission. Quite different results from the moving source fit were obtained in the $250 \mathrm{MeV}{ }^{16} \mathrm{O}+{ }^{116} \mathrm{Sn}$ reactions. As we can see from Fig. 2, a unique evaporative source fit is not sufficient to describe the experimental spectra for $\alpha$ particles. In the $250 \mathrm{MeV}{ }^{16} \mathrm{O}+{ }^{116} \mathrm{Sn}$ reaction, the large yields of nonequilibrium nature were observed both for the $\alpha$-particle and proton emissions. The ratio representing the total yield of pre-equilibrium particles divided by the total yield of evaporative particles and the results of experimental preequilibrium multiplicities are shown in Table 1. The large difference between the ratios for $\alpha$ particles and protons can be explained with the fact that the binding energy for $\alpha$ particles is smaller; therefore, it is easier to emit fast $\alpha$ particles. The fact that first-step $\alpha$-particle emission is more favorable than proton emission is normally supported by statistical model calculations for the thermalized emission.

The dominant process responsible for the pre-equilibrium emission is a pre-compound decay; however, another mechanism, namely projectile breakup, could also contribute to the final yield. In Fig. 2 one can see that a very small extra yield is still present in the $\alpha$-particle spectrum even after the double-source fit procedure. We had, therefore, to introduce a further source of production, that is again a fast emission source. In addition to a classical pre-equilibrium, 
$\alpha-$ spectra $500 \mathrm{MeV} \mathrm{Ni}+\mathrm{Zn}$
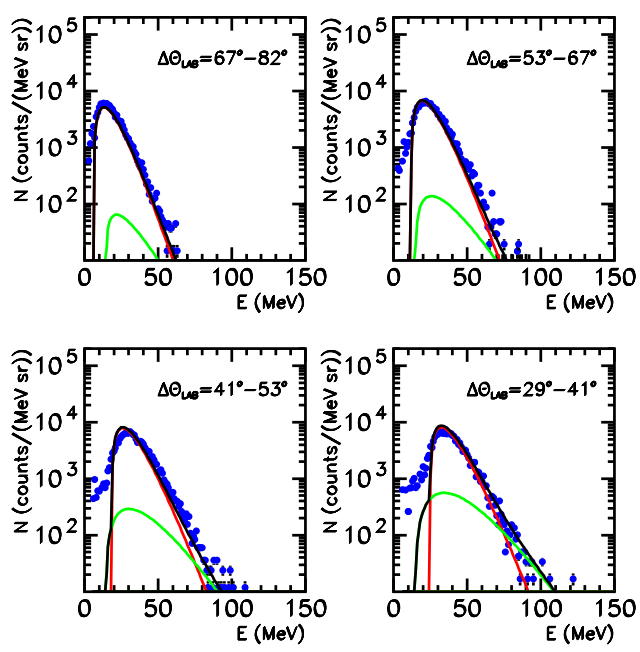

Fig. 1. Moving source fit (red line - evaporative source, green line - pre-equilibrium source, black line - sum of the two sources) for the experimental $\alpha$-particle laboratory energy spectra (symbols) for the $500 \mathrm{MeV}{ }^{64} \mathrm{Ni}+{ }^{68} \mathrm{Zn}$ reaction.

Table 1. The ratio of the total yield of particles from the preequilibrium source to the total yield of particles from the evaporative source $\frac{N_{2}}{N_{1}}$ and pre-equilibrium LCP Multiplicities $M^{P E}$. The error for the ratio $\frac{N_{2}}{N_{1}}$ is $10 \%$.

\begin{tabular}{llll}
\hline Reaction & LCP & $\frac{N_{2}}{N_{1}}$ & $M^{P E}$ \\
\hline $130 \mathrm{MeV}{ }^{16} \mathrm{O}+{ }^{116} \mathrm{Sn}$ & $\alpha$ & 0.21 & $0.05 \pm 0.01$ \\
$250 \mathrm{MeV}{ }^{16} \mathrm{O}+{ }^{116} \mathrm{Sn}$ & $\mathrm{p}$ & 0.30 & $0.25 \pm 0.03$ \\
$250 \mathrm{MeV}{ }^{16} \mathrm{O}+{ }^{116} \mathrm{Sn}$ & $\alpha$ & 1.30 & $0.22 \pm 0.02$ \\
$500 \mathrm{MeV}{ }^{64} \mathrm{Ni}+{ }^{68} \mathrm{Zn}$ & $\alpha$ & $\leq 0.1$ & $\leq 0.04$ \\
\hline
\end{tabular}

where the composite system emits neutrons and LCPs before it will be equilibrated, another source of the fast emission could be due to the projectile breakup. In our case, the oxygen projectile could break into a carbon and an $\alpha$ particle. The $\alpha$ particles are detected by the GARFIELD apparatus in coincidence with the ER resulting from the fusion of the remaining system $\mathrm{C}+\mathrm{Sn}$. Thus, in the $250 \mathrm{MeV}{ }^{16} \mathrm{O}$ $+{ }^{116} \mathrm{Sn}$ reaction we may say we observe three emission mechanisms which contribute to final yield, namely evaporative emission, pre-equilibrium emission and presumably projectile break-up

\subsection{Total CN Energy Loss}

The total composite system energy loss was determined using the following formula: $E_{\text {loss }}=\left(E_{k n}+E_{b_{n}}\right) \times M_{n}{ }^{P E}+$ $\left(E_{k p}+E_{b p}\right) \times M_{p}^{P E}+\left(E_{k \alpha}+E_{b \alpha}\right) \times M_{\alpha}^{P E}$, where $E_{k p}, E_{k \alpha}$ are the experimentally obtained average kinetic energies of the pre-equilibrium protons and $\alpha$ particles, respectively. $E_{b n}, E_{b p}$, and $E_{b \alpha}$ are the binding energies for neutrons, protons, and $\alpha$ particles, respectively. $M_{p}{ }^{P E}$ and $M_{\alpha}{ }^{P E}$ are
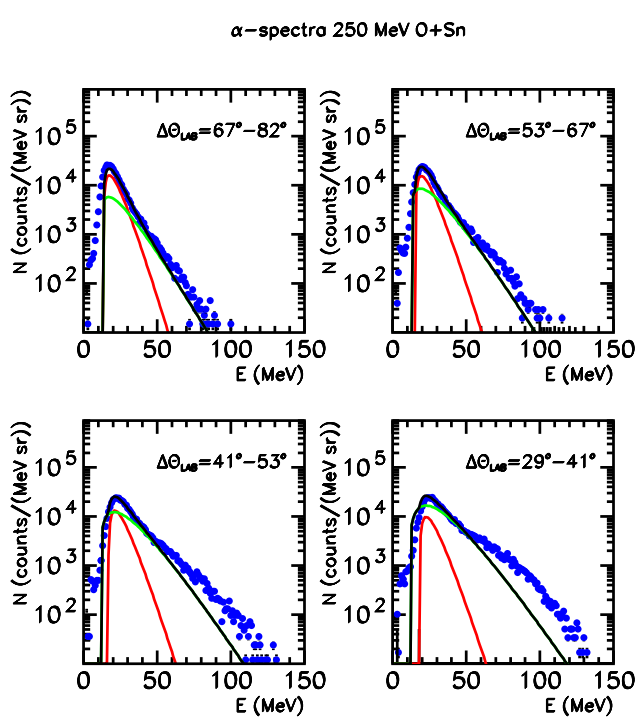

Fig. 2. Moving source fit (red line - evaporative source, green line - pre-equilibrium source, black line - sum of the two sources) for the experimental $\alpha$-particle laboratory energy spectra (symbols) for the $250 \mathrm{MeV}{ }^{16} \mathrm{O}+{ }^{116} \mathrm{Sn}$ reaction.

Table 2. Energy loss of the composite system formed in reactions ${ }^{16} \mathrm{O}+{ }^{116} \mathrm{Sn}$ and ${ }^{64} \mathrm{Ni}+{ }^{68} \mathrm{Zn}$ due to the pre-equilibrium particle emission in comparison with other experimental data (for reactions ${ }^{18} \mathrm{O}+{ }^{100} \mathrm{Mo}[3]$ and ${ }^{20} \mathrm{Ne}+{ }^{159} \mathrm{~Tb},{ }^{169} \mathrm{Tm}$ [5]) in the projectile energy region from 8 to $16 \mathrm{MeV} / \mathrm{u}$.

\begin{tabular}{llll}
\hline$E_{\text {beam }}, \mathrm{MeV} / \mathrm{u}$ & Reaction & $E^{*}, \mathrm{MeV}$ & $E_{\text {loss }}{ }^{P E}, \%$ \\
\hline 7.8 & ${ }^{64} \mathrm{Ni}+{ }^{68} \mathrm{Zn}$ & 203 & $3.6 \pm 2.0$ \\
8.1 & ${ }^{16} \mathrm{O}+{ }^{116} \mathrm{Sn}$ & 100 & $5.6 \pm 2.9$ \\
11.1 & ${ }^{18} \mathrm{O}+{ }^{100} \mathrm{Mo}$ & 174 & $21.4 \pm 2.6$ \\
13.0 & ${ }^{20} \mathrm{Ne}+{ }^{169} \mathrm{Tm}$ & 197 & $12.7 \pm 1.5$ \\
13.0 & ${ }^{20} \mathrm{Ne}+{ }^{159} \mathrm{~Tb}$ & 201 & $13.9 \pm 1.5$ \\
15.6 & ${ }^{16} \mathrm{O}+{ }^{116} \mathrm{Sn}$ & 206 & $19.9 \pm 3.4$ \\
16.0 & ${ }^{20} \mathrm{Ne}+{ }^{169} \mathrm{Tm}$ & 251 & $15.9 \pm 2.4$ \\
16.0 & ${ }^{20} \mathrm{Ne}+{ }^{159} \mathrm{~Tb}$ & 254 & $18.5 \pm 2.4$ \\
\hline
\end{tabular}

experimentally obtained pre-equilibrium multiplicities for protons and $\alpha$ particles, respectively. The pre-equilibrium neutron multiplicity $M_{n}{ }^{P E}$ which is one of the key parameters in the $\mathrm{CN}$ energy loss calculations was estimated from the systematic work of Cabrera et al. [5]. The average kinetic energy of the pre-equilibrium neutrons $E_{k n}$ was calculated using Griffin's exciton model (see Sect. 3.3).

The results of the energy loss of the composite systems formed in reactions $8.1 \mathrm{MeV} / \mathrm{u}, 15.6 \mathrm{MeV} / \mathrm{u}{ }^{16} \mathrm{O}+$ ${ }^{116} \mathrm{Sn}$ and $7.8 \mathrm{MeV} / \mathrm{u}{ }^{64} \mathrm{Ni}+{ }^{68} \mathrm{Zn}$ obtained in this work are shown in Table 2 together with other published experimental results in the projectile energy range from 8 to 16 $\mathrm{MeV} / \mathrm{u}$. Our results follow the general trend of the energy loss values obtained in [5]. Comparing the two reactions with mass-symmetric $\left(7.8 \mathrm{MeV} / \mathrm{u}{ }^{64} \mathrm{Ni}+{ }^{68} \mathrm{Zn}\right)$ and massasymmetric entrance channel $\left(15.6 \mathrm{MeV} / \mathrm{u}{ }^{16} \mathrm{O}+{ }^{116} \mathrm{Sn}\right)$ leading to the same composite system, we can confirm a 

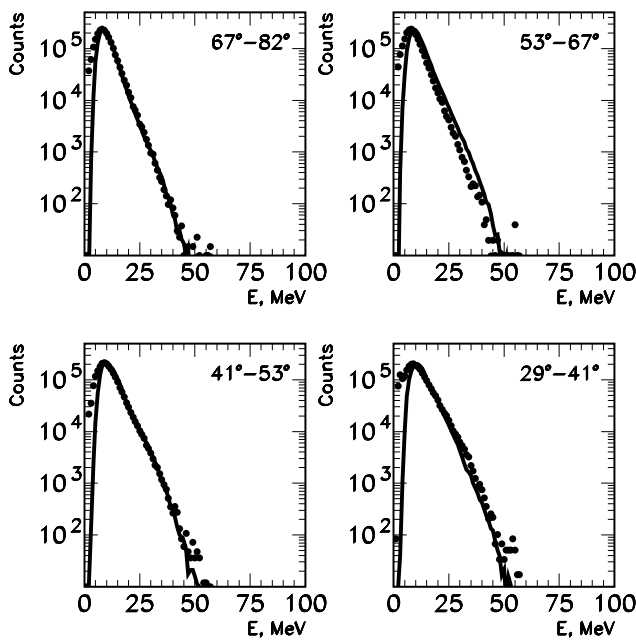

Fig. 3. Results of the model calculation (solid line) are shown together with the experimental proton laboratory energy spectra (symbols) for the $130 \mathrm{MeV}{ }^{16} \mathrm{O}+{ }^{116} \mathrm{Sn}$ reaction.

strong dependence of the pre-equilibrium emission on the projectile velocity.

\subsection{Model Calculation}

In the Griffin's exciton model [10], the state of a nuclear system produced by the collision between a bombarding particle and a target nucleus is determined by the exciton number $n=p+h$ and excitation energy $\mathrm{E}^{*}$. Here $p$ is the number of particles above the Fermi energy level and $h$ is the number of holes under the Fermi energy level. Among the free parameters of the model we vary the parameter $k$ of transition matrix element, the initial exciton configuration $\left(p_{0}, h_{0}\right)$, and the parameter $g$. The single particle level density $g$ around the Fermi surface is related with the level density parameter $a$ as $g=\frac{6 a}{\pi^{2}}$. The value $a$ is taken from the phenomenological model of nuclear level density [11]. The parameter $k$ defines the transition rates between the exciton configurations and time of the relaxation to the equilibrium state of the nuclear system. As a result, it affects the particle emission probability with energy $E_{b}$. The parameter $k$ is varied in the wide range from 100 to 800 $\mathrm{MeV}^{3}$. For more detailed description of the formalism we refer to section 4.1 of [12].

In the calculations of the pre-equilibrium neutron spectra we used the initial exciton configurations $n_{0}=(32 p, 1 h)$ and $n_{0}=(16 p, 0 h)$ for the ${ }^{64} \mathrm{Ni}+{ }^{68} \mathrm{Zn}$ and ${ }^{16} \mathrm{O}+{ }^{116} \mathrm{Sn}$ reactions, respectively, and the parameter $k=800 \mathrm{MeV}^{3}$. A first attempt in order to analyze experimental set of data was made for the reaction $130 \mathrm{MeV}{ }^{16} \mathrm{O}+{ }^{116} \mathrm{Sn}$ and the results are shown in Fig. 3.

\section{Conclusion}

Light Charged Particle spectra were measured in coincidence with heavy recoiling nuclei using a selective trigger on Evaporation Residues for different mass asymmetries in the entrance channel and different projectile energies $\left(300,400,500 \mathrm{MeV}^{64} \mathrm{Ni}+{ }^{68} \mathrm{Zn}\right.$ and $130,250 \mathrm{MeV}{ }^{16} \mathrm{O}+$ ${ }^{116} \mathrm{Sn}$ ). The experimental results were analyzed through a global moving source fit. In the case of mass-symmetric reactions, very small yields associated with the pre-equilibrium emission were obtained even for the highest energy case $\left(500 \mathrm{MeV}^{64} \mathrm{Ni}+{ }^{68} \mathrm{Zn}\right.$ reaction), while no pre-equilibriumemission yields were obtained for the ${ }^{64} \mathrm{Ni}+{ }^{68} \mathrm{Zn}$ reactions at 300 and $400 \mathrm{MeV}$. In the case of the mass-asymmetric reaction $\left(250 \mathrm{MeV}{ }^{16} \mathrm{O}+{ }^{116} \mathrm{Sn}\right.$ to be compared with the mass-symmetric reaction $500 \mathrm{MeV}{ }^{64} \mathrm{Ni}+{ }^{68} \mathrm{Zn}$ ), an evident contribution from a pre-equilibrium emission mechanism is found in the production yield. Furthermore, the analysis indicates the presence of a second fast emission mechanism. The second fast emission source seems to be associated with the $\alpha$-cluster structure of the oxygen beam and linked to the projectile break-up process. The energy loss of the mass asymmetric reaction $250 \mathrm{MeV}{ }^{16} \mathrm{O}+{ }^{116} \mathrm{Sn}$ was determined to be $40.9 \mathrm{MeV}$; therefore, the composite system is losing $19.9 \%$ of the initial excitation energy.

A small yield due to the pre-equilibrium emission was already observed in the reaction $130 \mathrm{MeV}{ }^{16} \mathrm{O}+{ }^{116} \mathrm{Sn}$ for $\alpha$ particles. From the comparison of the result obtained in this case $(8.1 \mathrm{MeV} / \mathrm{u}$ beam energy) with the upper limit of the pre-equilibrium $\alpha$-particle yield in the reaction 500 $\mathrm{MeV}{ }^{64} \mathrm{Ni}+{ }^{68} \mathrm{Zn}(7.8 \mathrm{MeV} / \mathrm{u}$ beam energy) we found that in the mass-asymmetric entrance channel case the preequilibrium to the evaporative yields ratio is at least two times larger. This confirms a dependence of the pre-equilibrium emission not only on the projectile velocity but also on the reaction entrance channel mass-asymmetry.

This campaign of experiments stimulated the continuation of the work in the research line concerning the development of the Griffin exciton model in a convolution with an evaporative statistical code.

\section{References}

1. D.A. Bromley, Treatise on heavy-ion science, vol. 2, (Plenum, New-York 1984)

2. M. Blann and B.A. Remington, Proceedings of the Texas AEGM Symposium on Hot Nuclei (College Station 1987) 145

3. M.P. Kelly et al, Phys. Rev. C 56, (1997) 3201

4. J. Gómez del Campo et al, Phys. Rev. C 60, (1999) 021601

5. J. Cabrera et al, Phys. Rev. C 68, (2003) 034613

6. M. Gonin et al, Phys. Rev. C 42, (1990) 2125

7. D.J. Hinde et al, Phys. Rev. C 45, (1992) 1229

8. A. Kumar et al, Nucl. Phys. A 798, (2008) 1

9. F. Gramegna et al, Nucl. Inst. Meth. 389, (1997) 474

10. J.J. Griffin, Phys. Rev. Lett. 17, (1966) 478

11. A.V. Ignatyuk, Sov. Journ. Nucl. Phys. 29, (1979) 875

12. D.O. Eremenko et al, Phys. Atom. Nucl. 65, (2002) 18 